\title{
ON CONSISTENT INTERGROUP COMPARISONS OF PURCHASING POWER OF MONEY
}

\author{
By M. Mukherjee and D. S. Prasada RaO \\ Indian Statistical Institute, Calcutta
}

\begin{abstract}
There is some advantage in comparing price levels using consistent methods because this gives unique results. This paper examines some available methods of consistent comparison, pointing out difficulties associated with heterogeneous data, and suggesting adaptations yielding better comparisons. Next, the problem of non-availability of price and quantity decomposition is considered. Another problem relating to non-identical lists of commodities and quality differences is tackled by using linear programming instead of regression methodology, both methods using some transformed variables. The procedure suggested is likely eventually to be useful for comparison of dissimilar countries. Computations on some Indian population groups illustrate the findings.
\end{abstract}

1. In this paper, we are concerned with a study of the problem of intergroup comparison of the purchasing power of money using consistent methods. The groups for our purpose are groups of population. They may be different countries or sections of the population within a country defined according to certain social and economic characteristics; they may also be the same country or a particular section within a country at different points of time. The notion of purchasing power of money may differ from one class of purchases to another, and we restrict our problem to the class defined by final expenditure in the sense the term is used in national income accounting. In actual examples, however, we use household consumption expenditure as a proxy for this. Normally, we consider not less than three groups for purposes of comparison. In this set-up, any particular group is characterized by a vector of prices, a vector of quantities and a vector of expenditures.

2. The problem of consistency: When not more than two groups are compared, the problem of consistency arises because unless special methods are used, different results are obtained when group $A$ is compared with group $B$ from when group $B$ is compared with group $A$. Thus, if $I_{a b}$ stands for the price index number of group $B$ with group $A$ as base, the normal procedures do not ensure that $I_{a b}=$ $1 / I_{b a}$. Even when this difficulty is resolved, a new difficulty arises when more than two groups $(A, B, C)$ are under comparison. In this case, normal procedures do not result in $I_{a c}$ being obtained as a product of $I_{a b}$ and $I_{b c}$. These, it will be observed, are the familiar time reversal and circularity tests discussed in the literature on index numbers. In our study of intergroup comparisons, we shall try to use index numbers that are consistent in this sense, and discuss their merits and deficiencies.

3. The problem here is to find $M$ numbers $\pi_{i}(i=1, \ldots M)$ for $M$ groups defining the price levels of the groups. If these numbers can be found, $I_{i j}$ is simply given by $\pi_{j} / \pi_{i}$. Obviously, many such sets of numbers $\pi_{i}$ can be found. For example, if all the groups are compared with one selected group, this would 
generate a consistent set. But, such consistent sets vary when the selected group used as base is changed. We may conveniently regard such sets as semi-consistent. The type of consistency we seek is such that it is independent of the selection of the base group. But even here, available methods may give more than one consistent set of index numbers and there is a choice between them.

4. In the ideal situation, we consider that all the groups have identical specifications for all the items included in the list of final expenditures. In this situation, the only problem centres around consistency, and one could get consistent systems of index numbers using different approaches. We may denote by $p_{i j}$ the price of $i$ th item in the $j$ th group and by $q_{i j}$ and $v_{i j}$ respectively the corresponding quantities and values.

5. In the Geary-Khamis approach $[1,8,10]$, we average prices of items given in rows, the price of the ith item $\left(P_{i}\right)$ coming as

$$
P_{i}=\sum_{j=1}^{M} R_{j} p_{i j} q_{i j} / \sum_{j=1}^{M} q_{i j} \quad(i=1,2 \ldots N)
$$

$R_{j}$ 's being some undetermined numbers representing purchasing power of money in different groups. Since, the purchasing power of money changes from group to group, it is necessary to introduce these numbers so that the values can be summed over groups. These numbers may be obtained from the $M$ equations,

$$
R_{j}=\sum_{i=1}^{N} P_{i} q_{i j} / \sum_{i=1}^{N} p_{i j} q_{i j} \quad(j=1,2 \ldots M) .
$$

The $N+M$ equations here are not independent, but it has been demonstrated that one non-trivial solution exists for $N$ prices and any $(M-1)$ purchasing powers in terms of the purchasing power of an arbitrarily chosen group, say the first $[13,14]$ under mild assumptions. Thus, we get a set of solutions $\left(R_{1}, R_{2} \ldots\right.$ $R_{M}$ ) when $R_{1}=1$, or in other words, we get a set of consistent price levels $\left(\pi_{1}, \pi_{2}, \ldots, \pi_{M}\right)$ when $\pi_{1}=1, \pi_{i}$ being equal to $1 / R_{i}$. It has been found empirically that the index numbers obtained in this manner are not widely different from the Fisher's Ideal index numbers based on adjacent pairs of ordinal groups of population formed according to their per capita aggregate expenditure $[6,12]$. While the individual differences are small, the Fisher's ideal system is inconsistent while the Geary-Khamis system is consistent.

6. In a method used by the Economic Commission for Latin America (ECLA), the consistency was achieved by averaging the quantities $\left(Q_{i}\right)$ instead of the prices first [5]. Here, we have

$$
Q_{i}=\sum_{j=1}^{M} q_{i j} / M \ldots(i=1,2 \ldots N)
$$

and with $\pi_{1}=1$,

$$
\pi_{j}=\sum_{i=1}^{N} p_{i j} Q_{i} / \sum_{i=1}^{N} p_{i 1} Q_{i} \ldots(j=1,2 \ldots M) .
$$

In this set-up, $Q_{i}$ 's and $\pi_{j}$ 's can be easily computed, and $I_{j k}$ is directly given by $\pi_{k} / \pi_{j}$. This is also a consistent system of index numbers. While the procedure is simple, the consistency here is based on an assumption that all the groups are 
governed by the same quantity vector. Since inter-country variations here may be large, this assumption appears to be unrealistic, or at least, less acceptable than those involved in the Geary-Khamis system. We have not paid any detailed attention to this method, though easy computability remains its obvious merit. It has also some symmetry with the Geary-Khamis method.

7. Another known method of obtaining a consistent system is due to Eltetö and Köves [9]. Let $F_{j k}$ stand for the Fisher's ideal index for the $k$ th group with the $j$ th group as base. Then $E_{j k}$, the corresponding Eltetö-Köves index number, is given by

$$
E_{j k}=\sqrt{F_{j k}^{(1)} \ldots F_{j k}^{(M)}}
$$

where

$$
F_{j k}{ }^{(l)}=F_{j l} F_{l k}, \text { for } l=1,2 \ldots M .
$$

It is easy to verify that $E_{j l c}$ is a consistent set of price index numbers. Further, they possess the property of being the only set of index numbers which minimises

$$
\sum_{j} \sum_{k}\left(\log E_{j k}-\log F_{j k}\right)^{2}
$$

subject to the consistency constraints. The Eltetö-Köves system thus provides a set of index numbers which are at a minimum distance from the Fisher's ideal index numbers, the distance being defined in a particular manner. However, the results are different and more complicated when simple Euclidean distances are used due to the non-linearities involved. It can be shown that $E_{j k}$ 's do not minimise the Euclidean distance from the Fisher's indices. It is of interest to note that the $E_{j k}$ 's coincide with the $E_{j k}{ }^{*}$ 's where

where

$$
E_{j k}^{*}=\left[\prod_{\substack{l=1 \\ p=1, l \neq p}}^{M} F_{j k, l p}\right]^{1 /[M(M-1)]}
$$

$$
F_{j k, l p}=F_{j l} F_{l p} F_{p k} \text { for } l, p=1,2 \ldots M .
$$

Here $F_{j k, t p}$ denotes the indexes obtained by the product of price changes from $j$ to $l, l$ to $p$ and $p$ to $k$. The Eltetö-Köves system probably has all the merits and deficiencies of the Geary-Khamis system. It has, however, an interesting feature in having a relation with the Fisher's ideal index numbers which are widely used for binary comparisons. It would be of interest to build up an Eltetö-Köves system based on a Geary-Khamis set of binary comparisons, giving a system likely to be more satisfactory than either Geary-Khamis system or the EltetöKöves system in view of certain desirable properties of the former in binary comparisons.

8. The attraction of consistent systems for intergroup comparison considered here is obvious. The solutions are based on the entire available evidence pertaining to the case and are unique. The main difficulty of the consistent system is that they do not satisfy some obviously desirable properties of index numbers. If in two groups under comparison, the prices in one group are uniformly higher than the prices in the other, a meaningful price index number is supposed to reflect this. Proportionality consistency [4] implies that a certain percentage 
increase in the prices of all goods should lead to a corresponding increase in the index number, and the property we consider here may be called a weak proportionality consistency requiring that if all price changes have a particular sign, the change in the index number should also have the same sign. The Geary-Khamis system possesses this property only when $M=2$ but not when $M>2$. This can be demonstrated by constructing counter examples showing that the index number does not have this property for $M>2$ [14]. This perhaps need not worry us very much when the expenditure patterns and the commodity composition of the groups are not widely divergent. But when very dissimilar groups are compared the risk may be considerable. One should also add here that when we desire a comparison between any two groups (A, B) out of $M$ groups, a simple binary comparison between $A$ and $B$ has to be better than any comparison using information about other groups. Likewise a binary comparison is also the best between groups $B$ and $C$. But apart from the question of inconsistency, it is not obvious that these two binary comparisons when linked provide the best possible comparison between the groups $(A, B, C)$ and nothing better could be done.

9. Multiple classification: So far, we have considered a uniform classification of groups or countries. For example, one may consider $M$ size classes of rural population (or $M$ countries). Supposing, however, we have $M$ size classes each for urban and rural areas, then the comparison between the $2 M$ groups can be made in different ways depending on prior judgement. We may, for example, consider the urban and rural areas as two larger groups having some special characteristics. In the case of countries, we can group them into categories having similar consumption patterns. After having done this, we may apply GearyKhamis or similar approaches within the larger groups and link the two groups through some selected size classes or countries one chosen from each group. The result in this case will be semi-consistent and yet different from that obtained when all the groups or countries are treated separately. There is also some reason to believe that the use of prior knowledge in selection of relatively homogenerous larger groups and of particular elements of groups for the purpose of linking, again from homogeneity considerations, may give better results than a straightforward application of Geary-Khamis or allied methods to all groups taken separately. Finally, the amount of computation necessary for obtaining the final results will be much smaller in this case. In the urban-rural problem, the largest matrices to be inverted will be $M \times M$ against the inversion of a matrix of dimension $2 M \times 2 M$ in the case of a straightforward application of GearyKhamis method.

10. We may now illustrate the method using the National Sample Survey (NSS) data relating to 1963-1964 collected in the 18th round of the survey, in which 21,721 rural and 25,978 urban households were used as the sample. We have 10 size classes each for rural and urban areas. Thus, the lowest decile for, say, rural areas comprises households supplying the poorest 10 percent of the population of rural India. We have taken 56 items of consumption for which price and quantity data are available. The prices in the NSS are computed by dividing the total expenditure on an item by the total quantity consumed. They obviously do not relate to items with fixed specification, being dependent on the composition of sub-items within an item. One can compare the 20 classes thus 
formed by using Geary-Khamis method in a straightforward manner. But instead, we may compare the rural size classes with the corresponding urban size classes and link these by using relative purchasing powers of size classes within the rural (or urban) area alone. Let us call comparisons within rural and urban areas as vertical and between urban and rural areas for the same size class as horizontal. ${ }^{1}$ Since in the general set-up, there can be $s$ groups (like urban and rural areas) for each size class $i$, we can make horizontal comparisons of the $s$ groups by using the Geary-Khamis method. Thus we calculate exchange rates $R_{i j}$ for $i=1,2 \ldots s$ and $j=1,2 \ldots t$ for $t$ size classes. Then we fix a group and make vertical comparisons, choosing the first group without loss of generality. This yields exchange rates $r_{i j}(j=1,2 \ldots t)$. Using the latter relation as link between the former relations, we define a price index number in the following manner. Consider two groups, say, $i_{1}$ th group in size class $j_{1}$ and $i_{2}$ th group in size class $j_{2}$. We measure the price change from $i_{1}$ th group in $j_{1}$ to the first group in $j_{1}$, from the first group in $j_{1}$ to the first group in $j_{2}$ and from the first group in $j_{2}$ to the $i_{2}$ th group in $j_{2}$, and define the price index number for group $i_{2}$ in $j_{2}$ with group $i_{1}$ in $j_{1}$ as base by

$$
I_{j_{1} j_{2}, i_{2} i_{2}}=\frac{R_{i_{1} j_{2}}}{R_{1 j_{1}}} \cdot \frac{r_{1 j_{1}}}{r_{1 j_{2}}} \cdot \frac{R_{1 j_{2}}}{R_{i_{3} j_{2}}}
$$

This definition involves the implicit assumption that the price change is multiplicative. The assumption is justified in the light of consistency tests like the time reversal and circularity tests. Further it can be verified that $I_{j_{1} j_{a}, i_{1} i_{2}}$ satisfies various consistency tests satisfied by index numbers constructed by GearyKhamis method. ${ }^{2}$

11. The application of the Geary-Khamis method to 20 fractile groups in a straightforward manner gives 20 values of exchanging rates $R_{i}(i=1,2, \ldots 20)$, the first 10 being for the rural groups and the following 10 for the urban groups, with $R_{1}=1$. In the procedure suggested, we compute, say, $10 R_{i}{ }^{*}$ 's for the rural size classes only with $R_{1}{ }^{*}=1$ and for each size class we obtain $R_{i}^{* *}$ for the urban area with the corresponding rural value taken as 1 . Thus, we get 20 figures comparable with earlier estimates based on the Geary-Khamis method, involving considerably less computation. It may be noted further that the figures are not widely different though the overall range of estimates reduces from 0.7077 to 1.0000 to 0.7668 to 1.0000 . All the possible price comparisons can conveniently be placed in a $20 \times 20$ matrix, the first 10 rows and columns showing the rural groups, the remainder showing the urban ones. The price index numbers based on our modified method, as can be anticipated, reduce the price variations between the extreme groups to some extent, though by and large, the figures are similar, in the sense that when the direct Geary-Khamis index shows a rise or a fall between two groups, the modified index also does the same in most cases. ${ }^{3}$

\footnotetext{
${ }^{1}$ When we have similar data for several groups over time, we may call the comparison between groups at a point of time as horizontal and the comparison within a group over time as vertical.

${ }^{2}$ Similarly, one may try to arrive at index numbers by calculating $R_{i j}(j=1,2 \ldots t)$ for each $i$ and $r_{i j}(j=1,2 \ldots s)$ for one $j$ and then define an index number similar to equation (6).

${ }^{3}$ It may be desirable also to try to link the urban and rural size classes by using one urban and one rural class having similar consumption patterns, after having estimates of exchange rates separately for all classes in urban and rural areas.
} 
12. Difficulties in respect of data: In applying any system of index numbers for an intergroup comparison, the set-up of full information which we have assumed so far has to be waived, at least partially, because we may not get all the information we need. Several types of data difficulties can be summarized as follows:

(i) It is frequently noticed that while the aggregate expenditure can be exhaustively broken down by a detailed list of items, it may not be possible to get separate quantity and price information for all the items. In view of this, attempts are sometimes made to obtain the expenditure pattern in an exhaustive way, and to obtain prices of some items in an expenditure category, for which exact specifications are provided. We thus get reasonably comparable prices, but they govern a small and unknown part of the expenditure category.

(ii) There are cases where the lists of expenditure categories are identical, but some items in the list for which price and quantity details are available have different specifications.

(iii) There may be cases when the lists themselves are not identical in the sense that they intersect only in respect of some of the items, the data in respect of expenditure, price and quantity being assumed to be available for all the common items. Thus, when $M$ groups are being compared there could be $n(n<N)$ items common to all groups; also between any two groups $i$ and $j$ there are $n_{i j}\left(n_{i j} \geq n\right)$ common items.

13. Confronted with a situation like this, it becomes necessary to adapt the index number systems so that we can make best possible use of all the available information, as well as information that can be collected within a reasonable time. It will be seen that some new methodological device can be used to tackle the problem, in addition to some proximate alterations of the systems already discussed. We shall consider these in the subsequent part of the paper.

14. Expenditures, but not their breakdowns, available for all items: When the share of expenditure contributed by the items which do not give price and quantity counterparts is small, the best course of action would be to work out $\pi_{i}$ 's on the basis of the items giving price and quantity breakdowns. Here the price level derived from the covered items is automatically applied to the items that could not be covered [6]. However, the procedure can be applied non-trivially to cover items without price and quantity components [13]. The expenditures categories can be lumped into several larger classes, each class having many items with price and quantity breakdowns and some items without such breakdown. These residual items can be lumped, and we may use here the price level derived from the items belonging to the class giving both price and quantity information. Since the classes can be formed so as to ensure sympathetic price movements of the covered and uncovered parts, on prior knowledge, the procedure suggested is an adaptation of the Geary-Khamis system for solving a problem that cannot be tackled by the system in its original formulation.

15. The problem can be illustrated using the NSS data considered earlier. We have only analysed the rural information here. Apart from 56 items of consumption used in the earlier example for which both price and quantity information were available, there was a residual group of items furnishing only expenditure data, the budget weight of the group increasing from 6.65 percent in 
0-10 group to as much as 35.56 percent in 90-100 group. Instead of obtaining the exchange rates on the basis of items which give full information, it is possible to classify these items into several use categories, and allocate the residual group of items to these use categories after having examined their characteristics. We can then obtain Geary-Khamis indexes for each class separately on the basis of the items with quantity and price details and let the expenditure shares in the class not covered in this manner be governed by these indexes. The overall index number comes out here as a weighted combination of the indexes of the several classes. In our example, the result of the procedure led to a slight increase in intergroup variability, the ordinal position of the group indexes remaining unchanged. This can be regarded as a non-trivial way of using information on items for which only expenditure data are available.

16. Normal procedure adopted for international comparison of dissimilar countries: In international comparison of dissimilar countries, the difficulty about information is formidable. The usual procedure here is to obtain expenditure vectors based on an identical list of items for a pair of countries $A, B$ going into a fair amount of detail, and then to choose some items with well defined specifications for which prices are available in both the countries to obtain the price relative, which governs the total expenditure of the category. This material is sufficient for comparing countries $A$ and $B$. When $A$ and $C$ are compared, however, the list of expenditure items may differ. In this way, all countries may be compared with $A$, providing what may be called a semi-consistent system. The system is semi-consistent because the results would differ when all countries are compared with a second country, say $B$. Also, while comparisons $(A, B)$ and $(A, C)$ would provide a link $(B, C)$, a direct $(B, C)$ comparison will certainly give a different result. Another difficulty of the method is the assumption that prices of some items within a common expenditure category meaningfully represent the prices of other items entering the category, in the chosen pair of countries. But the items for which specification prices are obtained may not contribute a large share of expenditure within a category. The assumption behind the procedure is that if we had price relatives for all the items within the category, the price relative we use is a weighted average of these. Whether this is approximately true or false has to be tested, at least for some categories, to convince ourselves of the validity of the general procedure. The investigation will involve collection of fresh data. In logic, the procedure is not strictly valid since the sets of items within a category will only partially intersect. Also, even for the common items, there could be quality differences. Next, it is not clear whether an a priori categorization of expenditures is desirable. Supposing we have price relatives covering $a$ percent of total expenditure in country $A$ and $\beta$ percent in country $B$, could not we choose categories a posteriori giving better results than a priori categorization? Finally, if we insist too much on exact specifications, $\alpha$ and $\beta$ will be small, and the overall comparison can be very much a result of the categorization system adopted, because one could think of another categorization system giving a different result. Incidentally, it becomes difficult in this method to assign meaning to quantities derived by dividing expenditures by prices. On the other hand, since expenditure vectors may differ from one comparison to another, the procedure enables us to use a larger volume of available data than in the case when we insist 
upon identical expenditure vectors for all countries being compared, as is required for consistent comparisons. Also, here the comparison between a pair $(A, B)$ is not altered by a new comparison $(A, C)$. The direct $(B, C)$ comparison is not attempted, because the corresponding expenditure vectors do not conform.

17. The survey we have considered earlier gives prices of different items of expenditure based on the entire information, obtained by dividing the values by the quantities. The expenditure items are formed in such a manner that addition of quantities for an item is meaningful in some sense. For rice, for example, there will be various qualities and the higher average price paid by the richer size classes is a result of the fact that these classes buy relatively more of fine rice while poorer classes buy relatively more of coarse rice. Nonetheless, if we suppose that rice meets some specific requirements (say, from the angle of supply of nutrients), adding together of quantities of fine and coarse rice is meaningful. In this sense, though more crudely, one could add quantities of different cereals, yardage of cloth, and so on. It is, therefore, not entirely meaningless to assume that the richer classes do pay higher prices for the same basic needs, and the type of comparison we have made has some sense. Had it been possible to cover sizable parts of expenditure categories by specification prices, that certainly would have been a better procedure. But so long as specification prices cover and could conceivably cover only small parts of the total expenditure of dissimilar groups, the procedure we have adopted may be tried side by side with that based on specification prices, to form a view about the relative purchasing powers of different dissimilar groups.

18. Regression method: Difficulties about specification prices can be bypassed in some cases by the application of what is known as the regression method [2,7]. The basic assumption here is that the price paid for a unit quantity of a particular commodity is for some measurable characteristics of the commodity. Thus, the price of beer depends not so much on the quantity of it but on the quantity of alcohol contained. The price of motor cars depends very much on the length, weight and horsepower of a car. Similarly, one could think of such measurable characteristics of various other commodities governing prices of unit quantities. Such hypotheses can be tested by regression analysis where price is the dependent variable and the various characteristics independent variables. When the analysis yields two regression equations in the two countries with high values of multiple correlation, these equations can be used in the following manner. For example, the dollar prices of Indian and American cars of average specifications obtained from the American equation give an index of quality and the actual price relative adjusted by the quality index gives a quality adjusted price relative between dollar and rupee for motor cars. The procedure thus enables us to obtain price ratios for cars even when American cars are rarely sold in India and Indian cars are not available in the U.S.A. Similarly, the Indian equation will yield another price relation between dollar and rupee. The procedure can be used extensively for other items for intercountry comparison as well as for intergroup comparison of the type we have been discussing. But the basic problem here is identifying measurable characteristics of this type for different commodities giving reasonably good regression equations. How far the procedure would succeed can only 
be judged when many more attempts have been made to derive the equations. This opens up a vast field of empirical analysis. But for the present, the procedure can be successfully applied only for a few items on the basis of the available studies. It is also not obvious that the regression approach should always lead to reasonable price relatives. Data may not give a good fit and the multiple correlation coefficients may turn out to be small. But the procedure suggests that it is fruitful to go beyond the quantities measured in customary units, and to try to find some basic characteristics. Such characteristics need not be only those that influence prices.

19. Programming approach: Since one aim of the price comparison between countries and groups is to obtain comparable average levels of living by deflating the aggregate expenditures by price index numbers obtained, we are permitted to seek such characteristics of commodities which directly contribute to the level of living, irrespective of the fact whether they have a close influence on the price or not. In other words, from the available quantity vectors, which may have some common and some non-overlapping items for a number of groups or countries, we try to proceed to a basic human requirement vector which has identical components for all groups or countries. In food, for example, the human requirements can be taken to be represented by caloric contents and basic nutrients. While not much attention has been paid to the subject, it is conceivable that for other items of expenditure also it would be possible to identify variables representing, in some sense, the basic human requirements. Once these variables are obtained, the problem is to find their prices, so that the intergroup or intercountry comparison of the purchasing power of money could be based on the prices and quantities of these new variables.

20. We associate a basic human requirement vector $b_{j}$ with $q_{j}$ for group $j$. Consumers spend $p_{j} q_{j}$ to obtain $b_{j}$. This implies that the individual pays a price for each of the elements of $b_{j}$. If we are able to obtain these prices $\left(y_{j}\right.$, say), then we have the vectors $y_{j}, b_{j}(j=1,2 \ldots M)$ for the $M$ groups. From this we can obtain a consistent system of index numbers by using Geary-Khamis or other methods. The problem can be solved by using linear programming in a way which avoids the difficulties associated with its application in index number problems. ${ }^{4}$

21. These difficulties can be overcome in the approach discussed below. Given $p_{j}$ and $q_{j}$ for the $j$ th group, we obtain the corresponding nutrient vector $b_{j}, b_{j}=A_{j} q_{j}$, when $A_{j}$ is the transformation matrix for the $j$ th group. ${ }^{5}$ Hence $p_{j}^{\prime} q_{j}$ can be viewed as the amount spent to acquire the vector $b_{j}$. If the individual

${ }^{4}$ In the usual application of the linear programming method to index number problems, the minimum expenditure necessary to attain the base period nutrient vector given the current period prices is obtained, and the ratio of this expenditure to the base period expenditure gives the required index. However, in this case, the index for a group with itself as base may be different from unity (i.e., $I_{j j} \neq 1$ ). This difficulty has been resolved in a paper by Balintfy and others [11]. Here optimal expenditures of different groups, necessary for obtaining a given nutrient vector, have been computed, the nutrient vector being decided in advance. Then the index numbers have been defined by respective expenditure ratios. But the method is not fully satisfactory because it uses an arbitrary nutrient vector and does not use the available quantity data.

${ }^{5}$ The transformation matrix needs change from group to group to take cognisance of quality differences, in a special sense. 
is assumed to be a cost minimiser, he should have spent the amount equal to

$$
\begin{aligned}
& \operatorname{minimum} p_{j}{ }^{\prime} q \\
& \text { subject to } A_{j} q \geq b_{j} \\
& q \geq 0
\end{aligned}
$$

This is true for all $j$. If the actual observations coincide with the solution, then only the individual is an optimiser, otherwise, we use the solution of this problem as a proxy for the actual quantity vector. Solvability of the above problem is easy to establish. Since $q_{j}$ is feasible for (7) and the feasible region is closed and bounded from below, the minimum is attained by the function $p_{j}^{\prime} q$ on the feasible set. We next consider the dual of the primal problem. In symbols, it can be written as

$$
\begin{aligned}
& \text { minimum } y_{j}{ }^{\prime} b_{j} \\
& \text { subject to } A_{j}{ }^{\prime} y_{j} \leq p_{j} \\
& y_{j} \leq 0
\end{aligned}
$$

The problem is also solvable by virtue of the fundamental duality theorem. The solution $y_{j}{ }^{*}$ has the conventional interpretation that they represent the "shadow prices" of various elements of $b_{j}$. This enables us to find a price for each of the nutrients $b_{j}$; further we have a legitimate claim for calling $y_{j}^{*}$ prices of $b_{j}$. Given these we can apply any procedure for constructing the price index numbers, ${ }^{6}$ using $y_{j}{ }^{*}$ and $b_{j}$ instead of $p_{j}$ and $q_{j}$.

22. Comparing this method with those devised by Balintfy and others, we find certain advantages. We are able to use the dual of the linear programming set-up to obtain economically meaningful "prices" of nutrients that are implicit in the system. Further, we avoid the difficulty of $I_{j j}$ being different from unity, make use of all the available information and do not depend on an arbitrary nutrient vector. On the other hand, we face the problem of zero prices for some of the nutrients in the solution of the dual. To tackle this, we change the dual problem slightly, and replace the constraints $y_{j} \geq 0$ by $y_{j} \geq \epsilon$ when $\epsilon$ is a positive vector with entries very near zero. The resulting programming problem can also be shown to be solvable.

23. Formidable difficulty still attaches to the procedure, particularly when it has to be applied to aggregate final expenditure. Considerable researches are needed to identify elements of the $b_{j}$ vector, that have to be homogeneous over $j$.

${ }^{6}$ Two observations about the index numbers derived from a linear programming approach of our type may be noted:

(1) If $p_{2}>p_{1}$, then the Paasche index from the shadow prices of nutrients and quantities of nutrients $P_{12}$ is greater than unity.

Proof: Let $y_{2}{ }^{*}$ and $y_{1}{ }^{*}$ represent the shadow price vectors of groups (2) and (1) respectively. Let the corresponding nutrient vectors be $b_{2}$ and $b_{1}$. Then by definition, $P_{12}=y_{2}{ }^{* \prime} b_{2} / y_{1}{ }^{* \prime} b_{2}$. Observe that $y_{1}^{*}$ is a feasible solution of the linear programming problem for group (2), for $A^{\prime} y_{1} \leq p_{1} \leq p_{2}$. Further $p_{2} \geq(1+\lambda) p_{1}$, for some $\lambda>0$. Hence, $y_{1}^{*}(1+\lambda)$ is also feasible for the problem of group (2). So, $y_{2}{ }^{* \prime} b_{2} \geq y_{1}{ }^{* \prime}(1+\lambda) b_{2}$ $>y_{1}^{* \prime} b_{2}$. Hence $P_{12}>1$.

However, only a weaker result is true in the case of Laspeyres index.

(2) If $p_{2}>p_{1}$ and $b_{2}$ is of the form $K b_{1}$, for $K>0$, then the Laspeyres index $y_{2}{ }^{* \prime} b_{1} / y_{1}{ }^{* \prime} b_{1}$ is greater than 1 . The proof is trivial. 
After having identified these, it is necessary to obtain transformation matrices $(A)$ to obtain $b$ 's from $q$ 's, and this would need substantial empirical work. Obviously, we need a lot of information other than price and quantity data to solve the problem. But on the positive side, apart from the advantages already pointed out, the method helps us to work with an identical list of basic requirements for all groups, and their associated prices, and there is little arbitrariness subsequent to this stage. Contrasted with the dependence on the information on the budget pattern and specification prices in respect of a small part of the total expenditure, in the case of international comparison of dissimilar countries, here we depend on the entire price and quantity information, a lot of subsidiary data and some clearly stated assumptions. It is admitted that we measure two different things in the two cases, but surely the intergroup or international comparison based on this method would be a meaningful one.

24. Confronted with difficulties about data, we illustrate the method by a simple example drawn from the statistical material already considered. In the example we consider some ordinal population groups (rural 80-90, 90-100 and urban 80-90 and 90-100) and a few important items of food (rice, wheat, milk, ghee, potatoes, meat and sugar) giving vectors $p_{i}$ and $q_{i}$ for each group. We then use a transformation matrix $A$ [based on 3], the elements $a_{i j}$ of which give the amount of the $i$ th nutrient in unit quantity of the $j$ th food item. Using this information and using the solution of the dual problem, we obtain vectors $b$ for nutrients and $y$ for corresponding shadow prices. These values have then been used to obtain the price index numbers based on the transformed variables. The important thing to observe about the results is that while calculations on original variables produce index numbers showing a somewhat higher intergroup variation, the pattern of intergroup variation depicted by the two systems is similar, suggesting that a linear programming approach of the type described can give a meaningful comparison of intergroup price variations. The basic results are given below:

Fisher's Ideal Index Numbers Based on Original and Transformed Variables

\begin{tabular}{|c|c|c|c|c|c|c|c|c|c|}
\hline & & \multicolumn{4}{|c|}{ Original } & \multicolumn{4}{|c|}{ Transformed } \\
\hline & & \multicolumn{2}{|c|}{ Rural } & \multicolumn{2}{|c|}{ Urban } & \multicolumn{2}{|c|}{ Rural } & \multicolumn{2}{|c|}{ Urban } \\
\hline & & $80-90$ & $90-100$ & $80-90$ & $90-100$ & $80-90$ & $90-100$ & $80-90$ & $90-100$ \\
\hline & & (1) & (2) & (3) & (4) & (1) & (2) & (3) & (4) \\
\hline \multirow[t]{2}{*}{ Rural } & $80-90$ & 1.0000 & 1.0240 & 1.2179 & 1.2532 & 1.0000 & 1.0116 & 1.1750 & 1.1935 \\
\hline & $90-100$ & & 1.0000 & 1.1850 & 1.2237 & & 1.0000 & 1.1672 & 1.1878 \\
\hline \multirow[t]{2}{*}{ Urban } & $80-90$ & & & 1.0000 & 1.0350 & & & 1.000 & 1.0176 \\
\hline & $90-100$ & & & & 1.0000 & & & & 1.0000 \\
\hline
\end{tabular}


25. Concluding observations: An attempt may now be made to analyse our findings with the aim of obtaining some guidelines for international comparison of dissimilar countries by using consistent methods. The semi-consistent method suffers from two defects. First, the insistence on specification pricing results in a very small part of the expenditure being governed by price data actually used, and second, adoption of a single base country leads to a doubt that the relative price levels thus obtained could change widely if other countries are used as bases. The discussions about the two difficulties, however, have to be at different levels.

26. If data do not permit anything but pricing a few items within an expenditure category, this price material can be utilized for a consistent comparison. This will admittedly involve using a somewhat nebulous quantity concept. We should note that the semi-consistent method has to depend on a nebulous quantity concept in any case. While imperfect, the quantity concept of the survey material used by us is perhaps better than this, but here the average price that the survey provides for an expenditure category in a group depends on the composition of commodities entering the category, and does not relate to any item or groups of items with exact specifications. Since, however, we have both price and quantity data for the entire range of transactions, the price concept is meaningful when the unit quantity involved can be supposed to cater to a specific human need. Since survey data of this type can be collected at reasonable cost, and also existing family budget statistics could be utilized for the purpose, comparison of a group of countries following this method does not appear to be entirely meaningless.

27. Since we can never hope to get prices with exact specifications to cover a significant part of the total expenditure, when dissimilar countries are being compared, it is reasonable to go beyond the quantities and prices of listed items, and to seek new variables linked with the quantities by some transformation matrices. The new variables may be chosen either for explaining prices itemwise as in the regression analysis or for representing some specific human needs globally, as in our programming approach. It is conceivable that after considerable researches, we arrive at a single list of items of basic human needs and their prices, enabling one to use a consistent method. This need not involve any additional logical difficulty beyond our agreeing to compare dissimilar countries. Admitting the possibility of such comparison probably implies postulation of the existence of such uniform needs.

28. We have observed that consistent methods may give funny results under special circumstances. But in spite of this, we feel that it will be desirable to base international comparison of dissimilar countries on some consistent method, because of the uncertainty about the results based on semi-consistent methods. Unless one can demonstrate that the system of price index numbers does not change widely with shifts in the base country, there will always be doubts associated with using the results. Such a demonstration will require much more data which could perhaps be used for obtaining a consistent system as well, and hence one may try to get a consistent system in any case.

29. It is difficult to pronounce clearly about the choice among the available consistent systems which can be applied on raw data. If we reject the ECLA approach due to reasons indicated, still there is a choice between the Geary- 
Khamis and Eltetö-Köves approaches. The latter has the advantage that it is clearly linked with the Fisher's ideal index while the former also has some theoretical attraction. It is our feeling that either of these two methods could be applied for actual calculations.

30. More systematic exploration is needed of the problem of classification of countries. Incidentally, since classification of countries into a few relatively homogeneous groups appears to be desirable in the light of our analysis, the consistent systems themselves will not give unique results, depending on classification systems adopted. In view of the fact that binary comparisons are known to be distorted by addition of new odd groups, we feel that grouping into homogeneous categories will be desirable. The index numbers for countries falling within different homogeneous categories can then be obtained by using a consistent method. The categories next can be linked by using a consistent method again on one country each picked out from each category. This selection should be purposive depending on homogeneity considerations. Some arbitrariness naturally would attach to such groupings and selection, but the rules used for the purpose should be discussed and agreed upon before the comparison is attempted. Finally, in all probability, the choice of the system of classification would have larger effect on the final results than the choice among the available consistent systems of index numbers,

\section{REFERENCES}

[1] Geary, R. C. (1958): A note on the comparison of exchange rates and purchasing powers between countries, J.R.S.S., Vol. 121, Part 1, 97-99.

[2] Griliches, Zvi (1961): Hedonic price indexes for automobiles: An econometric analysis of quality changes, Government Price Statistics, Hearings, U.S. Congress, Joint Economic Committee, p. 173-196.

[3] Chatterjee, G. S., Sarkar, D. and Paul, G. (1965): A preliminary note on the variation in levels of nutritional intake by household of rural India, Indian Statistical Institute (unpublished).

[4] Swamy, S. (1965): Consistency of Fisher's tests, Econometrica, Vol. 33, No. 3, p. 619-623.

[5] Braithwaite, S. N. (1968): Real income levels in Latin America, Review of Income and Wealth, Series 14, No. 2, June, p. 113-182.

[6] Mukherjee, M. (1969): A method of obtaining consistent price index numbers for interfractile comparison, 9th Indian Econometric Conference (mimeo).

[7] Kravis, I. B. and Lipsey, R. E. (1969): International price comparison by regression methods, International Economic Review, Vol. 10, No. 2. June, p. 223-246.

[8] Khamis, S. (1969): Neoteric index numbers, Stat/3/69, Indian Statistical Institute (mimeo).

[9] Eltetö, O. and Köves, P.: Egy nemzetkozi Osszehasonlitasoknel fellepo indexszamitasi problemarol (in Hungarian).

[10] Khamis, S. (1970): Properties and conditions for the existence of a new type of index numbers, Sankhyā, Series B, 32, Parts 1 \& 2.

[11] Balintfy, J. L., Nester, S. and Wasserman, W. (1970): Binary and chain comparisons with an experimental linear programming food price index, Review of Economics and Statistics, Vol. 52.

[12] Prasada Rao, D. S. and Mukherjee, M. (1970): On the comparison of heterogeneous groups in the Khamis-Geary method, 10th Indian Econometric Conference, 1970.

[13] Prasada Rao, D. S. (1971): On the existence and uniqueness of a new class of index numbers, Sankhya, Series B, Vol. 33, parts 3 and 4, p. 341-354.

[14] Prasada Rao, D. S. (1972): Contributions to the Methodology of Construction of Consistent Index Numbers, Ph.D Thesis, Indian Statistical Institute, Calcutta (unpublished). 